\title{
First Episode Mania at the Age of 78 Years: A Case Report
}

\author{
Dhonju G1, Singh PM², Joshi N¹
}

\author{
1. Junior Resident, Department of psychiatry, NMC, Kathmandu, Nepal 2. Asst. Prof. Department of psychiatry, \\ NMC, Kathmandu, Nepal
}

Email Corresponding Author: gunjanb2@gmail.com

\begin{abstract}
Late onset psychiatric disorders generate interest due to frequent association with some organic, neurological or metabolic etiology. Primary psychiatric illness with an onset in late life is rare. Late onset mania has not been frequently encountered and hence we are reporting a case of a 78 year old male diagnosed with first episode mania with psychotic symptoms.
\end{abstract}

Keywords: late-onset, mania, first episode

\section{INTRODUCTION}

Depressive episodes alternating with mania or hypomania represent the domain of bipolar disorders. The older figure of $1 \%$ for bipolar disorders in the general population is being challenged because of convincing data that these may account for $5 \%$ of the population and up to $50 \%$ of all depressions. Age of onset of bipolar disorder is mostly around 20 years, first-onset mania being rare among the elderly. The number of elderly with bipolar disorder is increasing because the elderly population is rising. ${ }^{1}$

\section{CASE REPORT:}

A 78 year old, male farmer, Hindu by religion, from a rural area, was brought to the outpatient by his family, with complaints of over talkativeness, boastfulness, irritability, aggressiveness, abusive behavior and speech, and disturbed sleep since 13 days. There was history of verbal altercation with a neighbor, regarding a financial matter, two days prior to the onset of illness. There was no history of head trauma, fever, unconsciousness, abnormal body movements, nausea, vomiting, altered urinary or bowel habits, alcohol or psychoactive substance use. He earned his living by making and selling a cultural threaded accessory- 'janai'. There was no significant history of prior medical or psychiatric illnesses, medications or illnesses in the family. $\mathrm{He}$ was well adjusted prior to the illness. General physical examination showed the vitals were stable and kyphosis was also noted. Neurological, cardiovascular, respiratory and abdominal examinations showed no significant abnormalities.

The Mental Status Examination presented a thin built elderly male, conscious, dressed appropriately for weather and culture, hygiene poor, increased psychomotor activity- pacing about, frequently visiting the female ward, spitting, talking in animated gestures, eye contact was maintained and sustained. Patient was not co-operative, rapport was difficult to establish. Speech was coherent, with increased rate, tone, volume and decreased reaction time. Mood was irritable and he showed pressure of speech, tangentiality, flight of ideas, grandeur delusions. No perceptual abnormalities were noted, he was oriented to time, place, person. Attention and concentration were aroused but not sustained, memory was intact. He had average intelligence, impaired judgment and insight was of Grade I.

The patient was admitted with a provisional diagnosis of Mania with psychotic symptoms, and investigations were sent. These included a random blood sugar, complete blood count, kidney function tests, liver function tests, VDRL, serum calcium, phosphorus and vitamin B12 levels which revealed no abnormalities, CT-scan head showed findings suggestive of age related cerebral atrophy and left maxillary sinusitis. Mini-mental state examination (MMSE) could not be done, he was uncooperative.

Since detailed clinical examination and investigations did not reveal secondary causes for the symptoms, the diagnosis of Mania with psychotic symptoms was made as per ICD-10 Classification of Mental and Behavioral Disorders. 
The patient was started on Haloperidol, increased to $5 \mathrm{mg}$ thrice daily, and trihexyphenidyl $2 \mathrm{mg}$ twice daily. Within ten days of admission, there was significant improvement of symptoms. MMSE was performed and the score was 27.The patient was discharged on same medication and followed up in a week, then on 2 weeks. Improvement was sustained and he was asymptomatic.

\section{DISCUSSION:}

Mania can be primary resulting from bipolar disorder or secondary resulting from pharmacological, metabolic, or neurologic causes. The etiology is important because although acute symptomatic treatment may be similar for both, appropriate treatment of secondary mania includes addressing the cause. ${ }^{2}$ The incidence of mania at age greater than 75 years is around 2 per 100000 persons. ${ }^{3}$ Late onset mood disorders, including mania, are often associated with vascular risk factors. ${ }^{4}$

In a critical review of bipolar disorder in elderly, fewer than $5 \%$ had first age of onset after 60 years of age. $^{5}$ In another review of 21 studies of older bipolar patients, the age of onset of mania was 56.4 years $(\mathrm{SD}=7.3$; range $38-70) .{ }^{6}$ In a study of patients with late-onset mania, the incidence of silent cerebral infarctions (SCI) was $65.0 \%$; which was significantly higher than for those with early-onset affective disorders and late-onset major depression $(P<.05) .{ }^{7}$ Various brain lesions reported as correlates of mania include bilateral orbito-frontal, right temporo-parietal, right basal, medial temporal, basal ganglia, thalamic and right fronto-temporal lesions. $^{2}$

Elderly patients with first manic episode exhibit increased cognitive impairment, although partially reversible. Cognitive dysfunction may suggest a diagnosis of dementia but there are differences in the presentations and pre-morbid histories. Agitation and psychosis in dementia typically occur later during the illness, rather than as initial presentation. ${ }^{8}$ Although substance abuse is often associated with younger adults, it must be ruled out in elderly with mania. ${ }^{2}$

A study on mood disorders in a geriatric clinic in India reported mean age of 68.5 years $(S D=5.75)$ in patients with mania at index visit. ${ }^{9}$ Two cases, a late onset secondary mania in a 61 year old with dengue fever and a first episode mania at 75 years were reported in India. 8,9
Pharmacological treatment for mania in the elderly is similar but with lower doses than for younger patients owing to their slower metabolism and sensitivity to side effects. ${ }^{5}$ Guidelines for younger patients cannot be extrapolated directly to elderly. It is important to study the efficacy and side effects of available treatments while taking into consideration the special characteristics of elderly patients. ${ }^{1}$ Even with treatment, bipolar elderly patients are at risk for limited, slow, or fragile symptomatic response. Since, cognitive impairment and recurrences are frequent, family-focused psychotherapy is important. ${ }^{10}$

Further studies are necessary for evaluation of bipolar disorders in elderly and formulation of appropriate management protocols. Geriatric bipolar disorder is becoming a growing public health problem and needs appropriate attention. ${ }^{5}$

\section{REFERENCES:}

1. Sadock BJ, Sadock VA, Pedro R. Kaplan and Sadock's Comprehensive textbook of Psychiatry, 9th Edn.Lippincott Williams and Wilkins, 2009, Vol 1, pp 1647-53.

2. Brooks JO, Hoblyn JC. Secondary mania in older adults. Am J Psychiatry 2005 Nov; 162: 11.

3. Kennedy N, Everitt B, Boydell J, Van Os J, Jones PB, Murray RM. Incidence and distribution of firstepisode mania by age: Results from a 35-year study. Psychol Med 2005; 35: 855-63.

4. Cassidy F, Carroll BJ. Vascular risk factors in late onset mania. Psychol Med 2002; 32: 359-62.

5. Depp CA, Jeste DV. Bipolar disorder in older adults: a critical review. Bipolar Disord 2004; 6: 343-67.

6. Goodwin F, Jamison K. Manic-Depressive Illness. Oxford: Oxford University Press, 1990.

7. Fujikawa T, Yamawaki S, Touhouda Y.Silent cerebral infarctions in patients with late-onset mania. Stroke 1995; 26: 946-9.

8. Tripathi SM, Mishra N. Late onset mania in dengue fever. Immunology and Infectious Diseases 2014; 2(1): 1-3.

9. Agrawal A, Kumar $R$, Sharma RC, Sharma DD. First episode mania at 75 years of age. Indian J Psychol Med. 2010 Jul-Dec; 32(2): 144-5.

10. Gildengers AG, Butters MA, Chisolm D, et al. Cognitive functioning and instrumental activities of daily living in late-life bipolar disorder. Am J Geriatr Psychiatry 2007; 15(2):174-9. 